\title{
Additional Immunohistochemistry Was Not Performed
}

National Cancer Institute

\section{Source}

National Cancer Institute. Additional Immunohistochemistry Was Not Performed. NCI

Thesaurus. Code C160310.

An indication that additional immunohistochemical tests were not performed during the study. 